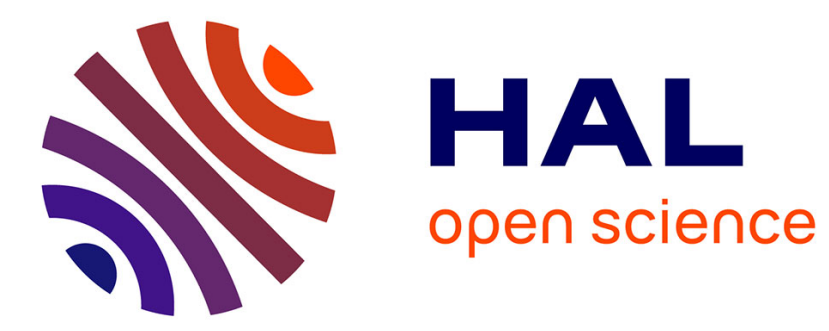

\title{
Développement professionnel. Variations et usages sur Internet.
}

\author{
Daniel Guy, Véronique Ortiz
}

\section{To cite this version:}

Daniel Guy, Véronique Ortiz. Développement professionnel. Variations et usages sur Internet. Travail et apprentissages: revue de didactique professionnelle, 2009, $\mathrm{N}^{\circ} 3$, pp.76 - 89. hal-01273812

\section{HAL Id: hal-01273812 \\ https://hal-univ-tlse2.archives-ouvertes.fr/hal-01273812}

Submitted on 14 Feb 2016

HAL is a multi-disciplinary open access archive for the deposit and dissemination of scientific research documents, whether they are published or not. The documents may come from teaching and research institutions in France or abroad, or from public or private research centers.
L'archive ouverte pluridisciplinaire HAL, est destinée au dépôt et à la diffusion de documents scientifiques de niveau recherche, publiés ou non, émanant des établissements d'enseignement et de recherche français ou étrangers, des laboratoires publics ou privés. 


\title{
Développement professionnel
}

\section{Variations et usages sur Internet}

\author{
Daniel GUY, Véronique ORTIZ \\ Université de Toulouse, CREFI-T¹. \\ guy@univ-tlse2.fr, veronique.ortiz@univ-tlse2.fr
}

Résumé : La question de départ interroge les variations et usages observables sur l'Internet du concept de " développement professionnel 》 dans des productions sociales diverses : chartres d'entreprise, offres de service, programmes de formation, articles de presse, textes réglementaires, communications scientifiques... L'objectif opérationnel est de caractériser les univers de référence sémantique des usages et d'analyser leurs correspondances pour mettre à jour l'acception du concept de développement professionnel et le système personnel - ou collectif - des valeurs donatrices de sens, c'est-à-dire l'ensemble des convictions et des croyances qui sous-tendent les usages sur Internet.

Mots clés : Développement professionnel - Usages - Internet - Carrière - Formation - Travail Emploi.

Abstract : The research questions the observable variations and uses on the Internet of the concept of "professional development" in various social productions: Chartres of company, offers of service, training programs, press articles, statutory texts, scientific papers... The operational objective is to characterize the universes of reference semantic of the uses and to analyze their correspondences to update the meaning of the concept of professional development and the personal system - or collective - values donors of direction, i.e. the whole of the convictions and beliefs which underlie the uses on the Internet.

Keywords : Development professional - Use - Internet - Digital environment - Career Formation - Work - Employment.

\footnotetext{
${ }^{1}$ Université de TOULOUSE, Centre de Recherche en Education, Formation et Insertion (CREFI-T) / Evaluation et Professionnalisation des Acteurs et des Contextes.
} 


\section{Introduction}

La question de départ de notre enquête interroge les variations et usages du concept de « développement professionnel ». Quelle idée du développement professionnel se forgent les acteurs engagés dans ses usages effectifs ? Pour répondre à cette question, il ne s'agit pas d'observer les usages sociaux à partir d'une conception systémique du développement professionnel élaborée dans le cadre d'un raisonnement abstrait articulé à un paradigme ${ }^{2}$. Il ne s'agit pas davantage, au terme d'une démarche inductive, de dégager des usages une conception opératoire du développement professionnel ${ }^{3}$. L'intention est plutôt de circonscrire l'idée du développement professionnel telle qu'elle s'exprime en propositions multiples dans des productions langagières francophones, mais élaborées indépendamment de toute intervention de l'observateur. Seront convoqués des chartres d'entreprise, des offres de service, des programmes de formation, des articles de presse, des textes réglementaires, des communications scientifiques... L'objectif opérationnel est de dégager les univers de référence sémantique du concept de développement professionnel et d'analyser leurs correspondances pour mettre au jour le système des valeurs donatrices de sens. Dans cette perspective qui reprend les bases théoriques de l'analyse sémantique conceptuelle, le concept, ce n'est pas le mot, pas même le mot clé, c'est en fait l'idée du locuteur telle qu'elle s'exprime en phrases multiples... C'est à la fois l'acception d'un mot clé et le système personnel - ou collectif - des valeurs donatrices de sens ${ }^{4}$. Dans ce contexte, les valeurs donatrices de sens renvoient à l'ensemble des convictions et des croyances à

\footnotetext{
${ }^{2}$ L'opposition entre concept opératoire et concept systémique est précisée dans le contexte de la construction d'un modèle d'analyse par Quivy R. et Campenhoudt L.-V. (1995). Le concept systémique n'est pas induit par l'expérience ; il est construit par raisonnement abstrait : déduction, analogie, opposition, implication, etc., même s'il s'inspire forcément des comportements des objets réels et des connaissances acquises antérieurement sur ces objets.

${ }^{3}$ Selon les mêmes auteurs, un concept opératoire isolé est - par opposition au concept systémique - un concept construit empiriquement à partir d'observations directes ou d'informations rassemblées par d'autres.

${ }^{4}$ Nous reprenons ici le projet de l'analyse sémantique conceptuelle proposée en 1967 par Klein M., Lantier F. et Ramognido N. mais dans une approche méthodologique renouvelée en appui sur les nouvelles technologies de l'information et de la communication.
} 
partir desquelles les acteurs - individuels ou collectifs - orientent leurs actions en élaborant une appréciation ou une estimation des situations auxquelles ils sont confrontés ${ }^{5}$.

\section{Dispositif et méthode de recueil des données}

\subsection{L'abondance des ressources numériques :}

En empruntant les multiples chemins du réseau Internet, le chercheur a la possibilité de naviguer d'un usage à l'autre, d'une entreprise de production de biens matériels à un laboratoire de recherche, d'un établissement de formation à une maison d'édition, d'un site gouvernemental à un blog personnel... De fait, l'exploration de l'espace numérique donne accès à un nombre important et varié d'usages : autour de 45000000 liens vers des occurrences en langue française étaient proposés en juillet 2008 par le moteur de recherche Google ; autour de 37600000 par l'annuaire Yahoo! France. Le panoramique est exceptionnel, la couverture suffisamment large pour caractériser les usages sociaux du concept de développement professionnel à travers l'analyse d'un échantillon de ses occurrences environnées de leur contexte numérique. En effet, au cours de la navigation sur Internet, l'observateur est confronté non seulement à des pages web rédigées et mises en forme spécialement pour nourrir le site d'une organisation, enrichir un blog ou contribuer à un forum, mais aussi à une part conséquente de l'ensemble des usages sociaux du concept de développement professionnel qui se retrouvent, à un moment ou un autre, versés sur Internet : textes réglementaires, accords d'entreprise ou encore articles scientifiques...

De plus, dans le cadre des formations universitaires au Conseil en développement professionnel, l'intérêt pédagogique de l'observation d'un corpus numérique ne cède en rien à son intérêt scientifique en invitant les étudiants «natifs du digital » à une approche méthodologique de la navigation sur Internet par l'exemple d'une recherche empirique, décrite avec précision ${ }^{6}$. Cette observation les aidera aussi à circonscrire parmi la globalité des usages sociaux, l'ensemble des

\footnotetext{
5 Jacques Ardoino et Guy Berger (1989) ont défini l'évaluation comme l'élaboration et la proposition d'une appréciation ou d'une estimation de quelque chose ou de quelqu'un. C'est, alors un processus (...) plus soucieux du sens et des significations, propres, particulières que de la cohérence ou de la compatibilité ou de la conformité à des modèles données.

${ }^{6}$ Dont l'université a pour mission de préparer leur insertion dans la société de la connaissance en réseau selon les termes du Rapport Isaac sur l'université numérique remis en octobre 2007.
} 
usages et variations professionnelles du concept de développement professionnel, étape essentielle d'une démarche d'orientation et d'insertion professionnelles? ${ }^{7}$.

\subsection{Le prélèvement de l'échantillon :}

Le prélèvement de l'échantillon paraît simple. D'une part, les données sont nombreuses, variées et accessibles. D'autre part, l'objectif est de saisir les usages sémantiques tel qu'ils s'expriment effectivement, sans critère de sélection a priori et sans connaissance préalable de leur distribution statistique. Au regard de ces éléments, le tirage aléatoire représenterait une solution optimale de prélèvement. Pourtant, cette technique n'a pas été retenue pour trois raisons. En premier lieu, l'ordre d'apparition des liens dans les résultats proposés par les moteurs de recherche ou les annuaires n'est pas lié au hasard : les résultats sont classés en fonction de leur pertinence ${ }^{8}$ supposée par rapport à la requête. En second lieu, les résultats ne peuvent orienter l'observateur que vers des ressources indexées, c'est-à-dire déterminées par une classification propre à l'annuaire consulté ou au robot de collecte et d'indexation des données. Enfin, les ressources liées ne sont pas toujours pertinentes et leur validation requiert l'intervention du chercheur. Faisant le deuil d'un échantillon aléatoire strict, nous avons opté pour une technique de sélection des données en dérivant sur la toile au fil des pages de résultats proposés par les moteurs de recherche, mais en traçant une route qui garantisse à la fois une représentation significative des sites nationaux ( $50 \%$ du prélèvement) tout en cherchant à privilégier la diversité des usages et des univers de référence. C'est pourquoi, chaque fois que nous avons rencontré des sites de pays peu représentés sur la toile, nous les avons sélectionnés, de même pour les usages ou les institutions. Concrètement, après une première exploration du réseau à l'aide des métamoteurs de recherche Kartoovisu, Ixquick et Seek.fr, nous avons sélectionné 180 ressources parmi les 50 premières pages de résultats en langue française indexés par le moteur de recherche EO-st.

\footnotetext{
${ }^{7}$ De développement professionnel diraient nos collègues québécois !

${ }^{8}$ Pertinence fondée pour partie sur la notoriété du site.
} 


\section{Description et caractérisation du corpus}

\subsection{Amplitude des unités de contexte :}

D'une occurrence à l'autre, l'amplitude de l'unité de contexte dans laquelle prend sens l'usage du concept de développement professionnel varie de quelques mots à plus d'une cinquantaine de lignes. Nous avons choisi de limiter la taille des unités de contexte sélectionnées à 1231 caractères espaces non compris, ce qui correspond au troisième quartile de la sélection opérée initialement par l'observateur'.

\subsection{Nationalité des sites indexés :}

L'observation du nom de domaine du site et/ou l'examen de son contenu permettent d'identifier la nationalité des sites hébergeant les unités de contexte sélectionnées. La catégorie « international » renvoie à des instances internationales de régulation comme l'ONU ou à des Organisations Non Gouvernementales. « Supranational » désigne des instances gouvernementales supranationales. C'est principalement le cas des instances européennes. Dans l'échantillon ${ }^{10}$, la présence des sites Canadiens a été minorée au profit de la représentation des sites européens, notamment nationaux. Ce choix traduit la volonté de circonscrire la zone d'emploi la plus probable des futurs cadres et conseillers du développement professionnel - notamment les étudiants de Master professionnel - formés dans les universités françaises sans pour autant faire l'impasse sur l'international, plus spécifiquement sur la référence majeure que représentent les usages canadiens.

\subsection{Indexation des contextes d'activités:}

Présentée en document annexe (A1), la table d'indexation des contextes d'activités dans lesquels prennent sens les usages numériques du concept de développement professionnel est le résultat d'une catégorisation a posteriori. S'écartant de la classification des organisations par secteur

\footnotetext{
9 Pour l'échantillon retenu : Min $=36 ;$ Max $=1231$

${ }^{10}$ Australie (1), Belgique (10), Bénin (1), Canada (48), Etats-Unis (3), France (90), Grande-Bretagne (1), Italie (1), Luxembourg (1), Serbie (1), Suisse (11), Thaillande (1), International (8), Supranational (4) pour un total de 180 ressources sélectionnées.
} 
d'activité économique, les catégories retenues ciblent les fonctions et les usages : l'éducation et la formation, le conseil et la consultance, le management et la gestion des ressources humaines, l'organisation et la régulation des professions, la recherche et la publication scientifique, la coopération internationale, les processus de fabrication et de développement de biens ou de services. Le signalement de la présence d'un lien de dépendance du site à une instance gouvernementale vient compléter la spécification du contexte d'activité.

\section{Les contextes sémantiques de référence}

\subsection{Dispositif et méthode :}

Pour dégager les contextes sémantiques de référence, la méthode prend appui sur le logiciel Tropes $^{11}$, notamment sur ses possibilités d'exploration rapide et de caractérisation des contextes sémantiques. Une analyse de contenu manuelle aurait pu donner des résultats assez proches, mais au prix d'un temps passé beaucoup plus important.

La méthode standard de Tropes privilégie la catégorisation du vocabulaire en fonction de classes sémantiques définies a priori par le scénario d'analyse standard. Néanmoins, Prenant en considération, tant la spécificité du contenu de la recherche que celle de la forme des textes en ligne - fréquente absence de linéarité, recours à des mots clés et à des pratiques discursives synthétiques - le scénario standard a été adapté a posteriori aux singularités du corpus. Afin d'éviter un brouillard sémantique produit par la multiplication des classes nécessaires au balayage d'un texte trop important, l'analyse porte sur 30 extraits tirés au hasard parmi les 180 sites sélectionnés.

Pour chaque classe du scénario d'analyse, la table sémantique présente la fréquence d'apparition, la définition en compréhension de son thème complétée par les principaux mots qui l'expriment dans le corpus (équivalents sémantiques). Nous observons ainsi, parmi les 30 extraits soumis à l'analyse (3755 mots), 112 mots équivalents à «l'enseignement, l'éducation et la formation », 60 au « travail et à l'emploi »... En complément de leur définition en extension et en

\footnotetext{
${ }^{11}$ Le logiciel TROPES est conçu selon les principes de l'analyse prédicative-propositionnelle (Ghiglione et al., 1998). Pour une première approche, voir : http://www.acetic.fr/tropesfr.htm, consulté en mai 2008.
} 
compréhension, le commentaire des classes s'appuie sur des unités de contexte extraites du corpus et caractéristiques des classes analysées. Arbitrairement et pour des raisons de place, leur nombre dans cette présentation des résultats est limité à trois.

\subsection{Classes sémantiques de fréquence supérieure à 20 :}

\begin{tabular}{|l|l|}
\hline 112 & $\begin{array}{l}\text { Enseignement, éducation et formation : école, élèves, enseignant, enseignement, études, } \\
\text { étudiant, formation, stage... }\end{array}$ \\
\hline 60 & Travail et emploi : travail, travailler, profession, emploi... \\
\hline 47 & Cognition et connaissance : connaissance, compréhension, pensée, stratégie... \\
\hline 37 & Institution et organisation : Institution, organisation, établissement... \\
\hline 36 & Catégories socioprofessionnelles : direction, patron, employé... \\
\hline 26 & Organisme: organisme, \\
\hline
\end{tabular}

Tableau 1 : classes sémantiques de fréquence supérieure à 20 pour $\mathrm{n}=3755$ mots

Le tri des classes sémantiques par ordre décroissant souligne la prégnance sémantique du monde de l'enseignement, de l'éducation et de la formation dans les contextes d'usage du concept de développement professionnel [tableau 1, ligne 1]. Toutefois, cette prégnance recouvre trois acceptions différentes du développement professionnel selon qu'il est appréhendé comme formation continuée ${ }^{12}$ (1), démarche d'orientation et d'insertion professionnelles (2) ou dans un registre proche de la professionnalisation (3).

1. Le développement professionnel comme formation continuée : Le développement professionnel continu (DPC) des enseignants - ou en cours d'emploi - est largement considéré comme une condition essentielle pour améliorer la qualité de l'enseignement et l'apprentissage des élèves. Il existe traditionnellement deux approches de DPC: les ateliers ponctuels de formation et la formation en cascade $^{13}$...

2. Le développement professionnel comme orientation et insertion professionnelles : Le Centre de développement professionnel et de placement en droit a été créé au début de l'année académique 2005-2006 à l'initiative de l'Association des étudiants en droit avec l'appui de la Faculté de science politique et de droit de l'UQAM. Il vise à répondre à un besoin exprimé depuis de nombreuses années par des générations d'étudiants, soit celui d'avoir à leur disposition une

\footnotetext{
12 Formation professionnelle initiale et / ou continue.

${ }^{13}$ http://www.id21.org/insights/insights-ed06f/art01.html, consulté en mai 2008
} 
ressource professionnelle pour faire le pont entre l'Université et le milieu juridique et qui serait axé exclusivement sur les carrières en droit ${ }^{14}$.

3. Le développement professionnel comme professionnalisation: Analyse des modalités de développement professionnel des enseignants en formation et au travail : L'offre de formation initiale et de formation continue proposée par l'IUFM a pour finalité globale la professionnalisation des enseignants.

Ce recours au concept de développement professionnel par des acteurs du monde de l'enseignement, de l'éducation et de la formation traduit leur conviction que la qualité des apprentissages et des enseignements est étroitement liée à des stratégies et à des dynamiques de formation et d'insertion à même de faire un pont entre l'université ou les instituts de formation et les milieux professionnels. Cette observation ne signifie pas que tous les acteurs du monde de l'éducation partagent cette conviction. Elle souligne que le réseau sémantique le plus prégnant parmi les usages du concept de développement professionnel renvoie au monde de l'éducation, en exprimant, dans cet univers, une position particulière.

Le monde lexical du travail et de l'emploi est, par ordre décroissant, la seconde catégorie sémantique de référence des usages du concept de développement professionnel [tableau 1, ligne 2]. Sa présence est d'autant plus nette qu'elle est renforcée par une catégorie de mots évoquant les catégories socioprofessionnelles comme direction, patron, employé, etc. [tableau 1, ligne 4]. L'observation de ces classes montre que les idées d'intégration et de carrière professionnelle cèdent le pas à celle d'insertion et de développement professionnel. D'un côté, une carrière garantie par la qualification. De l'autre, un développement professionnel articulé au modèle des compétences. Ce glissement de sens sur la relation d'emploi a déjà fait l'objet de nombreuses analyses ou travaux, notamment en sociologie du travail ou en sciences de l'éducation et de la formation. C'est par exemple le cas des travaux de Michel Fournet (2007) qui, à propos de l'autoformation des cadres européens de la net-économie, constate « que les entreprises déclarant ne plus pouvoir s'engager dans la durée (et) dans l'impossibilité d'assurer une carrière sur le long terme ne peuvent que promettre un développement des " compétences » monayables sur le marché de l'emploi et une rémunération individualisée de la performance (...). » Les traces de ce

\footnotetext{
${ }^{14}$ http://www.er.uqam.ca/nobel/cdpdroit/, consulté en mai 2008
} 
glissement dans le corpus sont assez nettes comme l'attestent la prise de position du Conseil international des infirmières (1), les pratiques de management chez Microsoft (3) ou encore les termes du protocole d'accord entre la Direction et les organisations syndicales sur les « facteurs d'avenir » à La Poste en France (3) :

1. La prise de position du Conseil international des infirmières (CII) : convaincu que le développement de carrière est un facteur important pour l'amélioration des systèmes de santé et des soins infirmiers dans le monde et qu'il contribue directement au maintien de la bonne qualité des services de soins. Par conséquent, le développement de carrière doit être encouragé et soutenu par un système d'enseignement structuré et par des structures de carrière reconnues (notamment une hiérarchie clinique) et suffisamment flexibles pour permettre la mobilité professionnelle et encourager l'exercice indépendant des soins infirmiers ou en profession libérale. Le CII soutient les travaux de recherche qui démontrent que la mobilité professionnelle a un impact positif : elle est en effet source de motivation pour le développement professionnel et d'une plus grande satisfaction professionnelle, ce qui garantit une couverture plus adéquate des services de santé $e^{15}$.

2. Les pratiques de management chez Microsoft : En mettant l'accent sur le développement professionnel de ses collaborateurs, Microsoft soutient l'acquisition de nouvelles compétences favorisant la professionnalisation des métiers et l'épanouissement de chacun. Deux moments forts jalonnent ainsi l'année de chaque collaborateur de Microsoft France :un entretien individuel avec son manager permettant d'évaluer ses performances sur la période écoulée et de fixer des objectifs pour la période à venir ; un entretien de développement professionnel, avec son manager qui constitue un moment privilégié pour faire le point sur ses compétences, envisager son développement sur le court et le moyen terme. Ces deux moments importants chez Microsoft sont accompagnés par les responsables en Ressources Humaines dédiés à chaque division. L'objectif est de faire coüncider les aspirations de développement professionnel des collaborateurs avec les enjeux de développement de Microsoft ${ }^{16}$.

3. Le protocole d'accord sur les "facteurs d'avenir »: La Direction du Courrier a initié un programme " Facteurs d'Avenir " visant à moderniser la distribution. Son but est de pérenniser et valoriser le métier et l'activité de facteur et de consolider un modèle social fondé sur l'emploi pérenne à temps complet. Le présent accord s'inscrit dans cette double volonté de valorisation et de consolidation. Il ne constitue pas, de la part des organisations syndicales signataires, une approbation explicite ou implicite des projets d'organisation de la Direction du Courrier et, notamment, du programme " Facteurs d'Avenir ". En revanche, dans le prolongement des "accords

\footnotetext{
15 www.icn.ch/pscardevf.htm, consulté en mai 2008.

${ }^{16}$ www.microsoft.com/france/travailler/rh/developpement/, consulté en mai 2008.
} 
de Vaugirard ", les parties considèrent qu'il convient d'assurer le développement professionnel des personnels de la distribution ${ }^{17}$...

Dans cette dernière unité de contexte, la tension entre l'idée implicite de carrière - consolider un modèle social fondé sur l'emploi pérenne à temps complet - et celle explicite de développement professionnel témoigne non seulement de l'âpreté des négociations et des enjeux liés à l'ouverture annoncée du marché national de la distribution, mais aussi du souci des partenaires sociaux de concilier la sécurisation, le perfectionnement et l'épanouissement des parcours professionnels individuels avec les exigences de flexibilité, de qualité et de productivité qui pèsent sur les organisations économiques. Cette volonté de conciliation prend sens dans un contexte sociopolitique plus général. Elle est ainsi au cœur du rapport sur la sécurisation des trajectoires professionnelles présenté au Conseil Economique et Social en 2007 par Madame Mme Édith Arnoult-Brill. De même, noterons en nous limitant à deux références essentielles pour étayer notre propos, l'Accord national interprofessionnel du 5 décembre 2003 relatif à l'accès des salariés à la formation tout au long de la vie professionnelle s'inscrit dans cette visée.

Les unités de contexte caractéristiques de la classe sémantique "Cognition et connaissance » associent étroitement le concept de développement professionnel au réseau sémantique du développement des compétences et des connaissances [tableau 1, ligne 3]. Mais cette association prend tout son sens, selon le corpus analysé, dans un environnement de gestion des ressources humaines caractérisé par le changement, la nouveauté. C'est le cas, par exemple, de la coopérative mondiale des bibliothèques nationales (OCLC) (1). De même, le groupe IBM exprime une préoccupation similaire afin d'attirer et garder les meilleurs candidats (2). Dans le domaine de l'éducation, Kirschner, P. \& Wopereis, I, (2003), en soulignant l'impact des technologie de la communication (TIC) sur le développement des communautés de pratique et sur la co-construction des connaissances nouvelles, participent de cet ancrage du concept de développement professionnel au réseau sémantique du développement des connaissances et des compétences (3) dans un contexte de changement et d'innovation, caractéristique des TIC. Développer ses compétences et acquérir ou construire de nouvelles connaissances pour s'adapter

\footnotetext{
${ }^{17}$ http://www.centre-inffo.fr/, consulté en mai 2008.
} 
au changement, tel est le discours conventionnel repris tout au long des unités de contexte environnant les mots clés de cette classe.

1. (OCLC) qui sait que le personnel des bibliothèques doit continuellement développer ses compétences et ses connaissances afin de relever les défis d'un environnement exigeant dans une ère où l'information évolue rapidement.

2. IBM se fait un point d'honneur d'attirer les meilleurs candidats, les plus intelligents. Elle leur garantit qu'ils pourront non seulement continuer à approfondir les connaissances qu'ils ont déjà acquises, mais également développer de nouvelles compétences tout au long de leur carrière.

3. Kirschner, P. \& Wopereis, I, (2003) sont de l'avis que les outils de communication encouragent et soutiennent les discussions et les discours, ce qui permet aux membres de la communauté d'interagir convenablement afin de co-construire de connaissances nouvelles. (p.113). Nous pouvons donc considérer que le développement professionnel des enseignants doit s'articuler autour des axes technologiques et sociaux.

\subsection{Classes sémantiques de fréquence supérieure à 10 :}

L'observation des classes sémantiques dont les fréquences d'occurrence sont comprises entre 10 et 20 permet d'élargir l'angle de vue en caractérisant les éléments périphériques du réseau sémantique dans lequel s'inscrit le concept de développement professionnel.

\begin{tabular}{|l|l|}
\hline 18 & Contrat : contrat, \\
\hline 17 & Communication et échanges : entretien, discussion, dialogue... \\
\hline 17 & Politique et gouvernance : Instances, gouvernement... \\
\hline 17 & Technologies et techniques :Techniques, technologies, nouvelles technologies... \\
\hline 16 & Argent et finance : financement, fonds et épargne, moyens de paiement... \\
\hline 13 & Projet : projet \\
\hline 13 & Action : action, activité, mise en œuvre... \\
\hline 12 & Groupes sociaux : les collaborateurs, les candidats, l'association des étudiants... \\
\hline 12 & Gestion : gestion, gestionnaire... \\
\hline 12 & Soutien : aide, soutien, entraide... \\
\hline 12 & Objectifs : objectif, but \\
\hline 11 & Innovation : innovation, création... \\
\hline
\end{tabular}

Tableau 2 : classes sémantiques de fréquence supérieure à 10 pour $n=3755$ mots 
Pour l'essentiel, ces classes sémantiques périphériques portent les traces du discours du management. Que ce soit tantôt à travers l'idée de projet, tantôt à travers celle de communication, de contrat de management, d'objectif, d'action, d'accompagnement, d'aide, d'innovation ou de changement, de gestion ou de nouvelles technologies comme en témoignent les illustrations suivantes :

1. Les activités de formation ont pour but le développement professionnel des stagiaires (individuels et équipes), l'accompagnement des changements, le développement des connaissances et pratiques liées aux projets des entreprises ${ }^{18}$.

2. Guide d'entretien professionnel (d'appréciation et de développement professionnel) : (...) L'entretien professionnel est avant tout un acte de management. L'entretien professionnel permet au salarié et à son hiérarchique de : Faire le point sur les réalisations, les difficultés, les besoins de performance ; Fixer de nouveaux objectifs pour la réussite de l'entreprise et des personnes qui la composent ${ }^{19}$.

3. Les actions liées à la formation et au développement professionnel découlent d'un processus de planification de la gestion des ressources humaines ${ }^{20}$.

Nous observons que les unités de contexte des mots clés caractéristiques de ce réseau sémantique associent à nouveau le concept de développement professionnel au croisement des objectifs pour la réussite de l'entreprise et des objectifs pour la réussite des personnes. Credo du « gagnant / gagnant »?

\section{L'analyse des correspondances}

\subsection{Dispositif et méthode :}

A l'aide du logiciel Tropes, nous avons balayé et décrit les univers sémantiques de référence des usages sociaux de l'idée de développement professionnel. Cette approche descriptive ne permet pas l'analyse multidimensionnelle des correspondances entre les différentes classes sémantiques d'une part, et entre les classes sémantiques et leur contexte d'usage d'autre part. C'est pourquoi, en appui sur le logiciel ALCESTE ${ }^{21}$, nous avons soumis à l'analyse des correspondances les 180

\footnotetext{
${ }^{18}$ www.conseil.com/formations_infres.html, consulté en mai 2008

${ }^{19}$ www.conseil.com/formations_infres.html, consulté en mai 2008.

${ }^{20}$ www.smq.qc.ca/publicsspec/smq/services/formation/, consulté en mai 2008.

21 Alceste - Analyse Lexicale par Contexte d'un Ensemble de Segments de Texte - est un logiciel d'Analyse de Données Textuelles, issu des travaux de Max Reinert.
} 
extraits de site sélectionnés initialement. L'étude statistique de la distribution du vocabulaire vise la mise au jour des univers de référence -mondes lexicaux- investis par les locuteurs. Une classification hiérarchique descendante (CHD) regroupe les unités de contexte élémentaires en fonction de la ressemblance / dissemblance de leur monde lexical de référence. La définition de variables illustratives $^{22}$, ici les contextes d'activités, permet de mettre à jour les correspondances entre le profil des classes concluant la CHD et les contextes d'usage. En complément de la CHD, une analyse factorielle des correspondances (AFC) effectuée sur le tableau croisant les formes lexicales significatives et leur classe d'appartenance permet d'affiner la description des correspondances.

\subsection{Les résultats de la classification :}

Pour interpréter les classes, l'analyste dispose d'un ensemble de résultats, notamment le dendrogramme, le profil lexical et les segments de texte caractéristiques de chaque classe. Le corpus des 180 extraits de sites en ligne est décrit à $76 \%$ par quatre classes stables qui regroupent 602 unités de contexte élémentaires.

\begin{tabular}{|c|c|c|}
\hline Des médecins (105 uce) & & \\
\hline Instances professionnelles & $\begin{array}{c}\text { Gouverner } \\
\text { Organiser } \\
\text { Réguler }\end{array}$ & \\
\hline Des avocats (77 uce) & \multirow[b]{3}{*}{$\begin{array}{c}\text { Conseiller } \\
\text { Accompagner } \\
\text { Conceptualiser }\end{array}$} & $\mathbf{V}$ \\
\hline Coaching et management (177 uce) & & \multirow{3}{*}{$\begin{array}{l}\mathbf{P} \\
\mathbf{R} \\
\mathbf{O}\end{array}$} \\
\hline Intervenants extérieurs & & \\
\hline Recherche et formation (243 uce) & & \\
\hline
\end{tabular}

Figure $1:$ Dendrogramme des classes stables

22 Les variables illustratives n'interviennent pas dans la détermination statistique des classes d'énoncés. En revanche, une fois les classes établies, leur degré de liaison à chacune des classes est calculé permettant ainsi leur mise en correspondance avec les univers lexicaux de référence. 
Les deux branches du dendrogramme différencient deux modèles du développement professionnel. L'un, endogène, prend principalement appui sur l'organisation interne de professions historiquement constituées, dont leurs instances de coordination et d'arbitrage comme l'Ordre des médecins ou le Barreau des avocats ${ }^{23}$. D'autres instances, moins connues, participent néanmoins de la même logique. C'est le cas, par exemple, de la Fédération Internationale des Associations de bibliothécaires et d'institutions dont l'objectif est clairement de protéger ses membres et de trouver des solutions à des problèmes mondiaux ${ }^{24}$ en favorisant la coopération internationale $^{25}$. Le second modèle relève d'une autre logique. Les vecteurs de développement professionnel ne sont plus endogènes mais exogènes selon deux modalités nettement différenciées.

* Dans le cas des entreprises ou du soutien individuel à des salariés, l'intervention prend la forme de l'accompagnement, du coaching ou du conseil. Des intervenants extérieurs aident l'entreprise, l'équipe de direction ou le salarié -principalement les cadres- à gagner en performance individuelle, s'affirmer dans le management d'équipe, mener à bien des négociations, résoudre un conflit, changer de métier, de fonction ou d'environnement ${ }^{26} .$.

- Dans le cas du développement professionnel des enseignants et des pratiques d'enseignement, le changement passe par une politique de formation et de soutien professionnels étayée par un travail de conceptualisation et d'analyse non seulement des objets en question, ici le développement et l'apprentissage professionnels, mais aussi des modalités de conduite du changement : quels appuis extérieurs ? quels types de recherche? quelles modalités de coopération et d'échange...

\footnotetext{
${ }^{23}$ Dubar et Tripier (1998) définissent les professions, dans leur caractéristique économique, comme des formes historiques de coalitions d'acteurs qui défendent leur intérêt en essayant d'assurer et de maintenir une fermeture de leur marché de travail, un monopole de leur activité, une clientèle assurée pour leur service, un emploi stable et une rémunération élevée, une reconnaissance de leur expertise. Définition reprise par Wittorski (2005).

24 On pensera notamment aux nombreux problèmes d'archivage, d'édition et d'accès aux ressources que pose le déploiement à l'échelle mondiale du réseau Internet. Cet exemple est intéressant car il montre comment une des plus anciennes institutions du savoir « les Bibliothèques » s’organise pour relever les défis de la modernité.

${ }^{25}$ http://www.cfifla.asso.fr/index.html, consulté en mai 2008.

${ }^{26}$ www.xineo.fr/Domaines d interventions.htm, consulté en mai 2008.
} 
Ce lien étroit entre les chercheurs et les praticiens n'est pas ignoré par les Ordres professionnels. Bien au contraire, que ce soit les exigences de formation qui pèsent sur les professionnels de santé afin d'être toujours à la pointe des avancées de la science dans leur domaine ou que ce soient les dynamiques complexes qui caractérisent le champ du droit en tension entre pratique et doctrine ${ }^{27}$. Cependant, dans le cas des pratiques enseignantes, l'école et l'université ne participent pas encore d'un ensemble fortement intégré comme dans le cas des professions de santé ou de droit. Même si la création des instituts universitaires de formation des Maîtres (IUFM) en France, et aujourd'hui leur réforme, semblent viser un rapprochement toujours plus grand entre ces deux mondes, la situation n'est pas comparable au degré d'intégration que réalisent les Centres Hospitaliers Universitaires (CHU) dans le domaine de la santé. Dans ce contexte, le chercheur apparait comme un acteur de changement relativement extérieur à l'Ecole au même titre que le consultant dans l'entreprise bien que les dispositifs de recherche et d'intervention conservent, chacun, leur spécificité.

\subsection{L'analyse factorielle des correspondances :}

$75 \%$ de l'inertie du tableau soumis à l'analyse ${ }^{28}$ est extraite par les deux premiers facteurs (sur un nombre total de 3). La figure 3 - dont la légende est présentée en note de bas de page ${ }^{29}$ présente la projection sur le plan F1.F2 des quatre classes stables concluant la CHD croisée avec la projection des variables illustratives caractérisant les contextes d'usage.

\footnotetext{
27 Pierre Bourdieu (1986) a montré dans «La force du droit » les effets d'exclusion du profane et donc de constitution et de renforcement des prérogatives d'un corps professionnel généré par la tension entre doctrine et pratique.

28 Pour mémoire, le tableau soumis à l'analyse croise les formes lexicales avec les quatre classes stables qui concluent la CHD. Effectif minimum d'un mot : 8 ; nombre de lignes analysées : 374 (fréq. mots $>8$ ) ; nombre total de lignes : 503 ; nombre de colonnes analysées (classes) : 4 .

29 Education, formation et insertion [EFI] ; Conseil, consultance, coaching et accompagnement [3CA] ; Management et gestion des ressources humaines [GRH] ; Organisation et régulation des professions [PROF] ; Recherche et publication scientifiques [RECH] ; Coopération internationale [COOPINTER] ; Processus industriel de fabrication et de développement de biens ou de services [DPI] ; Site officiel [GOUV].
} 


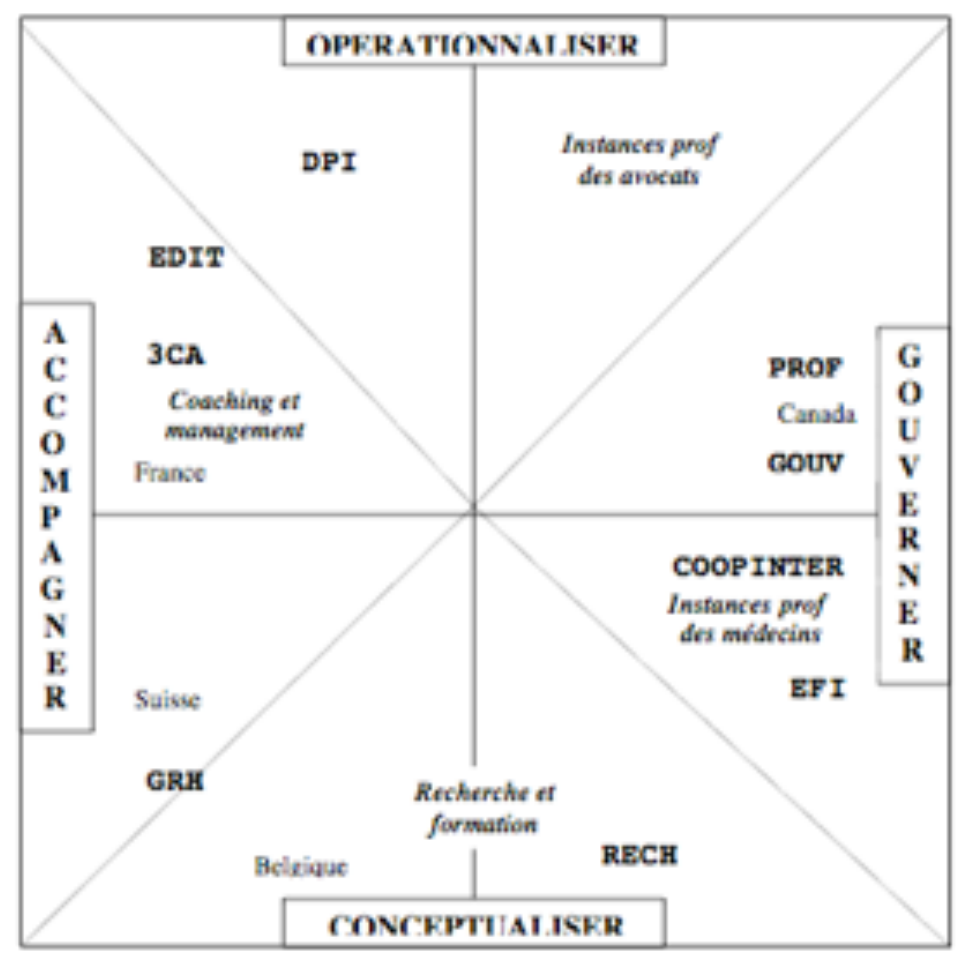

Figure 2 :Analyse factorielle des correspondances (F1.F2)

Le premier facteur différencie la gouvernance du développement professionnel de son accompagnement alors que le second met en regard conceptualisation et opérationnalisation. Dans cette projection, la gouvernance renvoie à l'ensemble des actions d'organisation, de régulation et d'arbitrage d'un champ professionnel : celui de la santé, du droit ou de l'éducation et de la formation. Cette gouvernance est non seulement le fait de décisions gouvernementales nationales ou supranationales, mais aussi le résultat d'un effort d'auto-organisation des professions à travers leurs instances de coopération, de concertation et d'arbitrage. A cette impulsion, cette coordination et régulation institutionnelles du développement répond dans le champ de l'acteur une conduite d'accompagnement, de soutien et de management du changement principalement mise en discours dans des contextes d'usage liés à la gestion des ressources humaines ou aux pratiques de conseil, de consultance, de coaching et d'accompagnement. Les contextes éditoriaux -hors publications scientifiques- mettent en scène un usage du concept résolument tourné vers son opérationnalisation notamment dans le cadre de l'accompagnement des acteurs qu'ils s'agissent de conseils pour favoriser le développement professionnel ou de témoignages sur le portfolio de compétences pour ne prendre que ces deux exemples. Les 
publications scientifiques sont centrées sur la conceptualisation et l'intelligibilité du développement professionnel alors que les offres de service de développement professionnel d'un produit soulignent leur expertise. Ce face à face entre opérationnalisation et conceptualisation ne renvoie pas à une ignorance réciproque de ces deux modes d'appréhension et de production du réel qui trouvent leur complémentarité dans une approche praxéologique du changement (F2), elle-même articulée à une visée politique du développement professionnel et de sa conduite (F1).

\section{En conclusion,}

L'objectif opérationnel de cette recherche était de caractériser les univers de référence sémantique des usages sociaux du concept de développement professionnel et d'analyser leurs correspondances pour mettre à jour l'acception de ce concept et le système personnel - ou collectif - des valeurs donatrices de sens, c'est-à-dire l'ensemble des convictions et des valeurs qui sous-tendent les usages. Ont été convoqués, via une navigation raisonnée sur internet, des chartres d'entreprise, des offres de service, des programmes de formation, des articles de presse, des textes réglementaires, des communications scientifiques... Dans le corpus observé, deux mondes sémantiques sont fortement représentés. Il s'agit d'abord de celui de l'enseignement et de la formation ; ensuite de celui - plus général - du travail, de l'emploi et des professions. Dans le monde de l'éducation et de la formation, le recours au concept de développement professionnel traduit la conviction que la qualité des apprentissages et des enseignements est étroitement liée à des stratégies et dynamiques de formation à même de faire un pont entre les instances de formation et les pratiques professionnelles en actes. Dans le monde du travail et de l'emploi, le concept de développement professionnel traduit le glissement de l'idée d'intégration et de carrière professionnelle à celle d'insertion et de développement professionnel. Les acteurs expriment alors le souci de concilier la sécurisation, le perfectionnement et l'épanouissement des parcours professionnels individuels avec les exigences de flexibilité, de qualité et de productivité qui pèsent sur les organisations économiques. De nombreuses traces attestent l'expression de cette conviction dans le corpus analysé. Il ne s'agit pas de dire ici que les pratiques de flexibilité ou d'amélioration de la productivité ont un impact positif observé objectivement sur le développement professionnel. Il s'agit de souligner que les usages accessibles via Internet du 
concept de développement professionnel traduisent la conviction que les antagonismes entre sécurisation et épanouissement des individus d'une part, et flexibilité et productivité des appareils de production d'autre part, peuvent être dépassés, les contraires conciliés, dans de nouvelles conceptions d'acquisition, de développement et de valorisation des compétences. Leurre idéologique ? A moins qu'en insistant sur la nécessaire corrélation entre le développement des acteurs individuels et celui des organisations, le concept de développement professionnel n'ancre le compromis que réalisent les professions entre le libéralisme et le protectionnisme, la tutelle et l'indépendance politiques, les garanties collectives et les responsabilités individuelles dans une forme politique particulière : la démocratie. John Dewey dans Reconstruction in philosophy, cité par Robert Horwitz (1987), rappelle que la principale signification de la démocratie, c'est le développement de chacun de ses membres: Le gouvernement, les affaires, l'art, la religion, toutes les institutions sociales ont un but. Ce but est de libérer et de développer les capacités des individus humains sans considération de race, de sexe, de classe ou de statut économique... Les matériaux recueillis, le questionnement initial et les résultats de notre enquête ne permettent pas d'approfondir notre investigation pour mieux spécifier et circonscrire le concept de développement professionnel dans la sphère de la philosophie politique même s'ils en ouvrent la voie en nous invitant à poursuivre la réflexion sur ce terrain.

\section{Bibliographie}

Ardoino J., Berger, G. (1989). D’une évaluation en miettes à une évaluation en actes. Le cas des universités. Paris : ANDASHA - Matrice.

Arnoult-Brill, E. (2007). Rapport sur la sécurisation des trajectoires professionnelles. Avis et Rapports du Conseil du Conseil Economique et Social, www.conseil-economique-et-social.fr/ rapport/doclon/07050612.pdf.

Bourdieu, P. (1986). «La force du droit », Actes de la Recherches en Sciences Sociales, 1986-09, nº4, p. 3-19. 
Dewey, J. (1950). Reconstruction in philosophy, New York : new american library, introduction, p.8-38.

Dubar, C., Tripier, P. (1998). Sociologie des professions, Paris : A. Colin.

Fournet, M. (2007). L'évaluation du processus d'autoformation des cadres européens de la netéconomie in A. Jorro (ED.), Evaluation et développement professionnel. Paris : L'Harmattant, $35-53$

Ghiglione, R., Landre, A., Bromberg, M., Molette, P. (1998). L'analyse automatique des contenus. Paris : Dunod.

Horwitz, R. (1994). Dewey, in Histoire de la philosophie politique, édition Leo Strauss et Joseph Cropsey. Paris : PUF, col. Leviathan, p. 945-964. History of political philosophy, University of Chicago Press, 1962, 1972, 1987.

Isaac, H. (2007). Rapport à Madame Valérie Pécresse, Ministre de l'Enseignement Supérieur et de la Recherche. L'université numérique en libre consultation sur le site http:// www.recherche.gouv.fr.

Kirschner, P. \& Wopereis, I.G.J.H. (2003). Mindtools for Teacher Communities : a European perspective in Technology Pedagogy and Education, Vol. 12, N¹, (p.105-123).

Klein, M., Lantier, F., Ramognido N. (1967). Une analyse sémantique conceptuelle. Bulletin d'études et recherches psychologiques, 1967 - XVI - n³, 275-287.

Quivy, R., Campenhoudt, L.-V. (1995). Manuel de recherche en sciences sociales. Paris : Dunod.

Wittorski, R. (sous la direction). (2005). Formation, travail et professionnalisation, Paris : Editions l'Harmattan. Collection : Action et Savoir.

\section{Document annexe}

\section{Table d'indexation des contextes d'activité :}

Pour chaque entrée, la table présente sa désignation, son code, son poids dans l'échantillon et une illustration extraite du corpus. 


\section{Education, formation et insertion $[\mathrm{EFI}][\mathrm{n}=44]$}

Le service national du récit en développement professionnel a pour mandat de favoriser le développement de compétences chez les enseignants et les élèves par l'utilisation pédagogique des TIC (technologies de l'information et des communications) $)^{30}$.

\section{Conseil, consultance, coaching et accompagnement $[3 \mathrm{CA}][\mathrm{n}=39]$}

EGoPrism est un outil de développement professionnel crée pour permettre à l'individu de retrouver ses repères en lui-même, et aider à la prise de décision dans un environnement mobile en permanence ${ }^{31}$.

\section{Management et gestion des ressources humaines [GRH] [n=22]}

Entièrement dédié au développement professionnel des collaborateurs, l' Espace RH vous accompagnera dans votre orientation professionnelle que ce soit en terme de formations, comme en terme d'écoute et de suivi individualisé32.

\section{Organisation et régulation des professions $[\mathrm{PROF}][\mathrm{n}=22]$}

Le contrat de développement professionnel intérimaire (CDPI) doit permettre à un salarié intérimaire, peu ou pas qualifié, d'accéder à un premier niveau de qualification ou à une qualification différente, voire complémentaire, permettant de multiplier ses opportunités d'emploi. Dans un souci d'égalité de traitement avec les salariés permanents et afin d'optimiser la construction des parcours professionnels des intérimaires en valorisant le travail effectué au cours des missions ${ }^{33}$...

\section{Recherche et publication scientifiques $[\mathrm{RECH}][\mathrm{n}=19]$}

Enquête internationale sur les programmes d'initiation et de développement professionnel destinés aux parlementaires. Ce document vous a été remis parce que vous êtes le Secrétaire général de votre Chambre I Parlement. L'UIP et l'Université Monash mènent cette enquête afin de savoir quels programmes d'initiation ou de développement professionnel ont été proposés aux parlementaires de votre chambre, élus ou nommés pour la première fois suite aux élections législatives les plus récentes.

\section{Coopération internationale $[$ COOPINTER $][n=9]$}

\footnotetext{
${ }^{30}$ http://www.recitfp.qc.ca/spip.php?article1, consulté en mai 2008.

${ }^{31}$ http://www.egoprism.com/intro, consulté en mai 2008.

${ }^{32}$ http://tf1rh.fr/page.php?page=3, consulté en mai 2008.

${ }^{33}$ http://www4.centre-inffo.fr/v2/cpnfp/NT328661.phtml, consulté en mai 2008.
} 
Bourses de développement professionnel. Objectifs : promouvoir et renforcer l'expertise individuelle et collective dans les pays en développement afin de favoriser leur autonomie et les encourager à appliquer leurs expériences locales en matière de développement au profit ${ }^{34}$.

Processus industriel de fabrication et de développement de biens ou de services [DPI] $[\mathrm{n}=8]$ "Quatre D" met à votre disposition un environnement intégré permettant d'accélérer le cycle de développement et de déploiement de vos applications. Au fil des années, 4D a enrichi les fonctionnalités de ses produits pour offrir un environnement de développement professionnel à ses clients, tout en conservant la facilité de prise en main des outils $R A D^{35} \ldots$

\section{Site officiel [GOUV] [n=20]}

Un contrat d'égalité professionnelle (CEP) est une mesure financière créée en 1983 (loi Roudy) afin d'inciter les entreprises à faire progresser l'égalité professionnelle entre les hommes et les femmes. A l'initiative d'Alberte SKORIC, responsable du centre de développement professionnel, et de Chrystel CALLIES DE SALIES, déléguée départementale aux droits des femmes et à l'égalité, ce contrat d'égalité professionnelle a été signé officiellement le 15 avril 2008 en présence de membres du service public départemental de l'emploi, de Jean-Yves MORACCHINI, sous-préfet de Thonon-les-Bains, de Dominique DAURES, déléguée régionale aux droits des femmes et à l'égalité, des maires de Publier et de Thonon-les-Bains ${ }^{36}$.

\footnotetext{
${ }^{34}$ http://www.scholarships.gc.ca/oas/oas-dev-fr.html, consulté en mai 2008.

${ }^{35}$ http://www.4d.fr/corporate/press/pdf/corporate-brochure-FR.pdf., téléchargé en mai 2008.

${ }^{36}$ http://www.haute-savoie.pref.gouv.fr/, consulté en mai 2008
} 